\title{
MODELLING OF THE HUMAN KNEE JOINT SUPPORTED BY ACTIVE ORTHOSIS
}

\author{
V. MUSALIMOV and Y. MONAHOV \\ Department of Mechatronics \\ National Research University of Information Technology, Mechanics and Optics \\ Saint-Petersburg, RUSSIA
M. TAMRE, D. RÕBAK, A. SIVITSKI, G. ARYASSOV and I. PENKOV*
School of Engineering, Tallinn University of Technology
Ehitajate tee 5, 19086 Tallinn, ESTONIA \\ E-mail: igor.penkov@ttu.ee
}

\begin{abstract}
The article discusses motion of a healthy knee joint in the sagittal plane and motion of an injured knee joint supported by an active orthosis. A kinematic scheme of a mechanism for the simulation of a knee joint motion is developed and motion of healthy and injured knee joints are modelled in Matlab. Angles between links, which simulate the femur and tibia are controlled by Simulink block of Model predictive control (MPC). The results of simulation have been compared with several samples of real motion of the human knee joint obtained from motion capture systems. On the basis of these analyses and also of the analysis of the forces in human lower limbs created at motion, an active smart orthosis is developed. The orthosis design was optimized to achieve an energy saving system with sufficient anatomy, necessary reliability, easy exploitation and low cost. With the orthosis it is possible to unload the knee joint, and also partially or fully compensate muscle forces required for the bending of the lower limb.
\end{abstract}

Key words: knee joint, modelling, active orthoses, biomechatronics.

\section{Introduction}

Rehabilitation process after an injury of a lower-limb is very important for every patient. A lot of researchers have tried to model and optimize the rehabilitation equipment. The one group of biomechanic lower-limb and pelvic constructions consists of models based on inverted pendulum models [1]-[4]. The next group of mathematical models consists of multilink constructions simulating human lower-limb motion [5]-[7]. The third group of lower-limb mathematical models is composed of models created on the basis of captured data from sensors during real experiments of human walking [8]-[18].

Knee joint is one of the most complex and vulnerable joints in the human musculoskeletal system and one of the most difficult joints from the point of biomechanics [19] due to articulating the longest levers of the lower limb: the femur and tibia [20]. The knee joint is a movable connection of the femur, tibia, fibula and patella. Meniscus, capsular-ligamentous apparatus and muscle-tendinous complexes are also included in the knee joint. Figure 1 shows an ordinary knee joint where 1 and 7 are collateral ligaments, 5 and 6 are circular ligaments, 2 is the tibia, 3 is the fibula, 4 is the femur, and 8 is the patella [21].

Almost every person in an explicit or implicit form faces injuries of the knee joint. For rehabilitation after a knee joint injury different kinds of orthosis are usually used but choosing an optimal orthosis is a long and laborious process. The model, proposed in this research, makes it possible to simplify the choice of orthosis and to avoid possible complications.

\footnotetext{
${ }^{*}$ To whom correspondence should be addressed
} 
There are a lot of models for the simulation of the knee joint on various levels, depending on research. It can be [22]-[26]:

- a two-dimensional planar mechanism with four or six links,

- a model, where three successive rotations of the knee joint are considered,

- a model with six degrees of freedom, where bone's surfaces were obtained by direct measurement,

- a model of the knee joint, supported by a passive orthosis.

Despite the variety of the models, a study on knee joints supported by an active orthosis can be found very seldom.

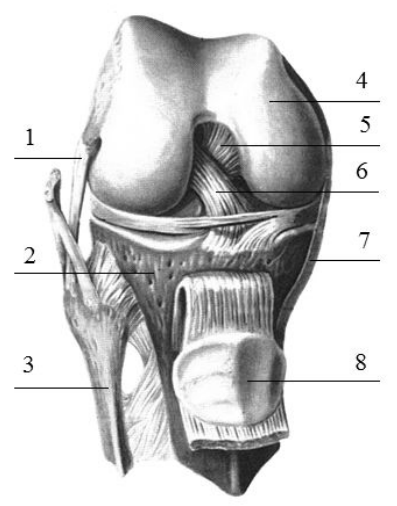

Fig.1. Right knee joint [22].

A lot of studies consider development of exoskeletons, components, and housings [27]-[31]. One of the applications of these systems is to assist people with disabilities, but the disadvantages include a long period of training, low autonomy and high cost of the orthoses. Active orthoses are being developed at many universities [32], [33] and some of the devices have already been patented [34]. However, unfortunately such devices are still not available on the consumer market.

The purpose of this research is to develop a model of an active orthosis, to analyze motion of the knee joint and to design a smart orthosis. The main objective of the device is to unload the injured knee joint, partially or fully compensate muscular effort required to bend the lower extremities, and also to restore the moving functions during the rehabilitation period. The resulting device will compensate the load applied by the human body on the knee joint during the movement. The linear actuator will restore the flexion of the knee joint. The autonomy of the device ensures its simple usage and maintenance, and due to the flexibility of settings, it would be easily adjustable to each individual patient.

\section{Motion capture for getting and analyzing of experimental data}

Kinematic and dynamic parameters describing human motion were obtained by an optical system consisting of ten Bonita cameras. The experiments were carried out at the St. Petersburg Institute of Cinema and Television. Each person from the experimental group performed several tasks, such as walking in a circle and in a straight line. Locations of the markers for the optical system are shown in Fig.2a, (point 4 is on the back of the leg). The inertial system consists of three accelerometers gy-61 and the board Arduino Mega. The location of the sensors on the leg is shown in Fig. $2 \mathrm{~b}$.

Data obtained by the inertial system for point 7 (Fig.2) are shown in Fig.3. The yellow line shows the acceleration measured by accelerometers, the red and blue lines represent velocity and displacement obtained by integration of acceleration and velocity accordingly. Since the data obtained by the capture system are very close to the integrated data, the tests were performed on the basis of the capture system because in this case no integration errors arise. 


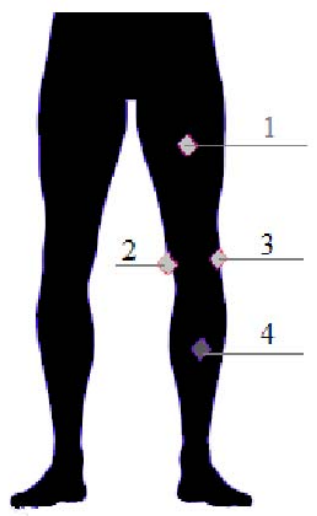

a)

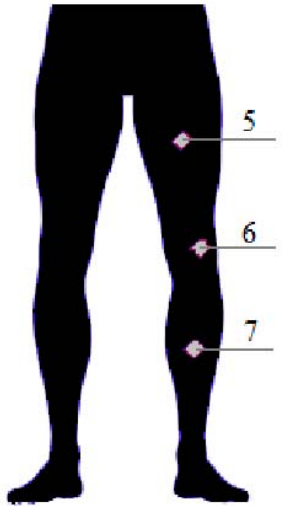

b)

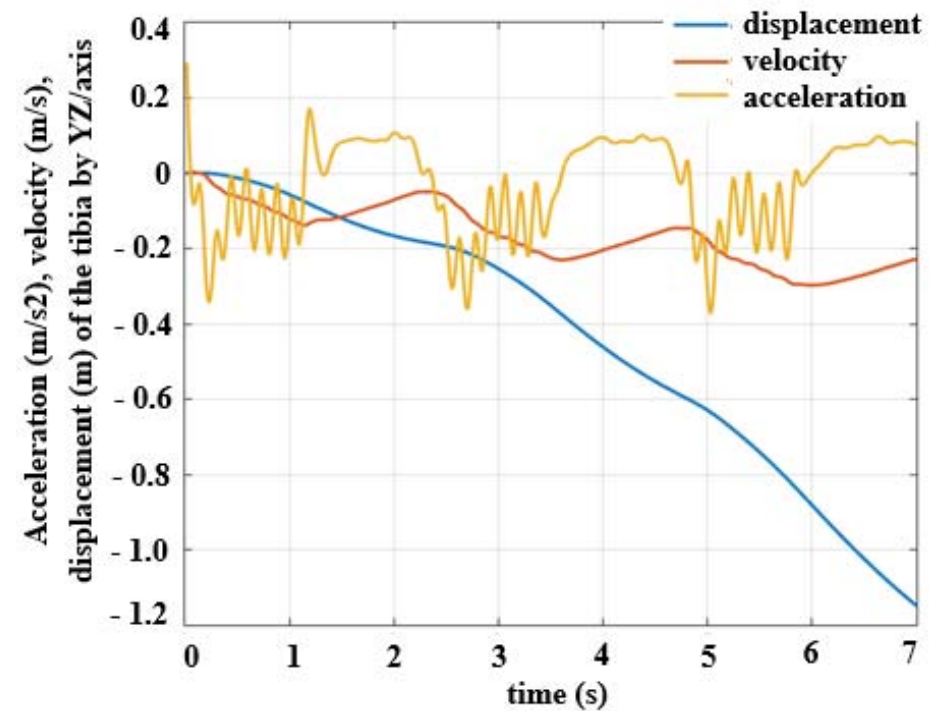

Fig.2. Location of the markers and accelerometers. a - location of the optical system's markers, $\mathrm{b}$ - location of the accelerometers.
Fig.3. Acceleration, velocity and displacement of the point 7 (Fig.2).

yellow line - acceleration, red line - velocity, blue line - displacement.

\section{Kinematics of a knee joint model}

On the basis of the results, a model of the knee joint will be created but before it is necessary to chart a mechanism allowing motion in the sagittal plane. The model should be applied to test various designs of the active orthosis of the knee joint. Structurally, the model is based on the average data of the bones dimensions and a simplified representation of the mating surfaces of the bones.

A kinematic scheme of a mechanism simulating a knee joint is shown in Fig.4. It is a planar mechanism with one degree of freedom. The length of the links simulating bones and other parameters of the mechanism corresponds to the parameters of an average person (height $-175 \mathrm{~cm}$, mass $-70 \mathrm{~kg}$ ), but all these parameters can be changed in accordance with the parameters of an individual patient [35], [36].

In this scheme (Fig.4), link 8 represents the tibia, link 5 represents the femur, these two links simplistically imitate connected surfaces which form the knee joint. Movement of the link which imitates the tibia around the link, which means the femur, is simulated by links that imitate the cruciate ligament. Link 6 imitates the anterior cruciate ligament and link 7 imitates the posterior cruciate ligament. Point $\mathrm{L}$ of this mechanism is the instantaneous center of velocity. Link 4 imitates the patella and patella's ligament, its corrected motion is provided by link 3 . Links 1 and 2 imitate quadriceps and tendon of quadriceps.

Let us assume that surfaces of the femur are two rigidly interconnected spheres (link 5), and the tibia surface is a plane (link 8). Surfaces of the femur will slide over the tibia surface. The static Cartesian coordinate system is associated with the femur. The origin of the coordinate system (point $\mathrm{O}$ ) is located equidistant between two centers of spheres. The $\mathrm{OX}$ axis is perpendicular to the sagittal plane, the $\mathrm{OZ}$ axis is directed left, and the OY axis is directed so that the OXYZ coordinate system is right-handed. The equation of the surfaces of two rigidly interconnected spheres at the OXYZ coordinate system can be written as:

$$
\begin{aligned}
& (x-a)^{2}+y^{2}+z^{2}=R^{2}, \\
& (x+a)^{2}+y^{2}+z^{2}=R^{2} .
\end{aligned}
$$




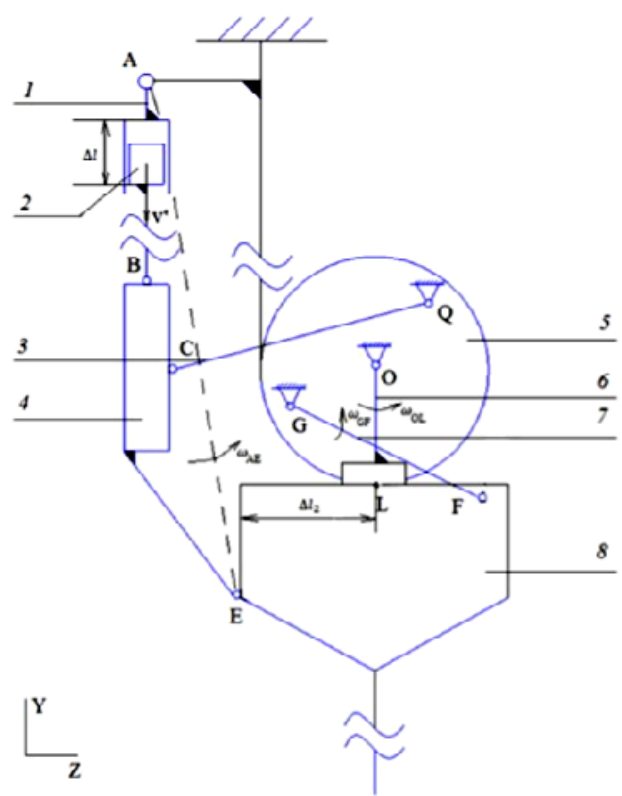

Fig.4. Mechanism for simulation of a knee joint motion at the sagittal plain.

Let us consider links simulating circular ligaments: link 6 - the anterior cruciate ligament, and link 7 - the posterior cruciate ligament. These links impose limitations to links 5 and 8 . It can be written as

$$
\begin{aligned}
& \left(Z_{F}-Z_{G}\right)^{2}+\left(Y_{F}-Y_{G}\right)^{2}=G F^{2}, \\
& \left(Z_{L}-Z_{O}\right)^{2}+\left(Y_{L}-Y_{O}\right)^{2}=L O^{2}, \\
& \left(Z_{F}-Z_{L}\right)^{2}+\left(Y_{F}-Y_{L}\right)^{2}=\left(L F+\Delta l_{2}\right)^{2} .
\end{aligned}
$$

The patella (link 4) is considered as a disk with radius $R_{l}$ and thickness $H\left(H=0.75 * R_{l}\right)$. The disk is rigidly connected to the rod, simulating the lower part of the patella ligament. The rod is connected to link 2 by a flat joint. Link 4 is movably connected to link 3 and link 3 is connected to link 5 . The limitations imposed by these links can be written as

$$
\begin{aligned}
& \left(Z_{Q}-Z_{C}\right)^{2}+\left(Y_{Q}-Y_{C}\right)^{2}=Q C^{2}, \\
& \left(Z_{B}-Z_{C}\right)^{2}+\left(Y_{B}-Y_{C}\right)^{2}=B C^{2}, \\
& \left(Z_{E}-Z_{C}\right)^{2}+\left(Y_{E}-Y_{C}\right)^{2}=E C^{2}, \\
& \left(Z_{E}-Z_{B}\right)^{2}+\left(Y_{E}-Y_{B}\right)^{2}=E B^{2}, \\
& \left(Z_{E}-Z_{F}\right)^{2}+\left(Y_{E}-Y_{F}\right)^{2}=E F^{2},
\end{aligned}
$$




$$
\left(Z_{E}-Z_{L}\right)^{2}+\left(Y_{E}-Y_{L}\right)^{2}=\Delta l_{2}^{2}+L E^{2}+\frac{\Delta l_{2}^{2} \cdot\left(L E^{2}+L F^{2} E F^{2}\right)}{L F} .
$$

Besides of bones and ligaments, the quadriceps (links 1 and 2) was also modeled. The quadriceps is considered as an extensible link. It also imposes some limitations on the mechanism that can be presented as

$$
\left(Z_{B}-Z_{A}\right)^{2}+\left(Y_{B}-Y_{A}\right)^{2}=(A B+\Delta l)^{2}
$$

\section{Matlab models of the knee joint}

\subsection{Model of the healthy joint}

Before modeling the motion of the knee joint supported by orthoses, it is necessary to create a model simulating the motion of a healthy knee joint [37]. This model is represented in Fig.5.

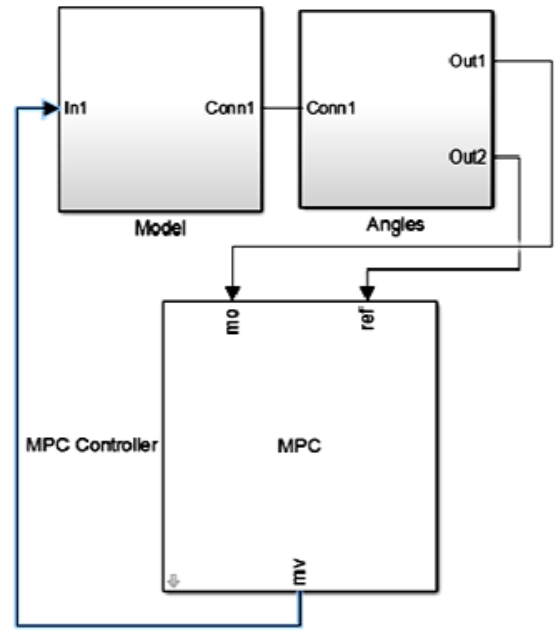

Fig.5. Model of a healthy knee joint.

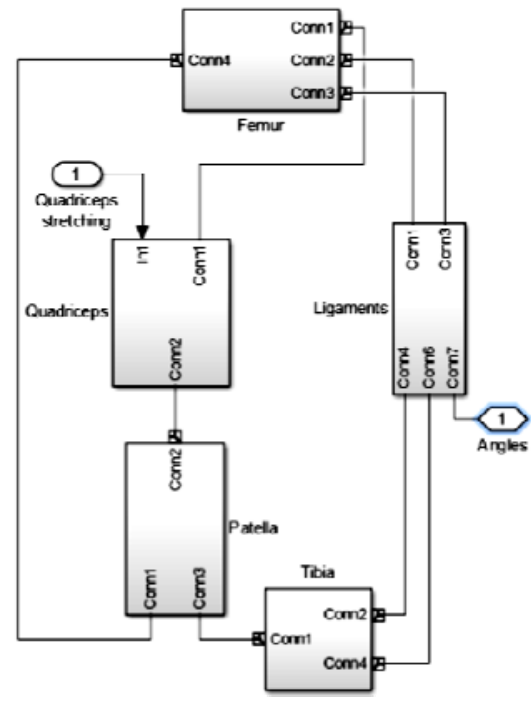

Fig.6. Block "knee joint model".

Angles between the tibia and the femur should correspond to the real angles, therefore it is necessary to simulate forces in the quadriceps correctly. Mathematical calculations of dynamics are automated and performed using the MPC block (Matlab/Simulink). This block controls links simulating the quadriceps (links 1 and 2) and angles between the tibia and the femur.

For modeling, it is necessary to know the model parameters: body weight, length of links, inertia moments, and the mutual arrangement of links. All these parameters are given in model subsystems; each subsystem simulates a certain part of the knee joint.

The femur subsystem (Fig.7) simulates a femur $0.46 \mathrm{~m}$ long and with the radius equal to $0.03 \mathrm{~m}$. In the model being discussed, the femur subsystem will be considered as the base of the mechanism. The quadriceps subsystem (Fig.8) consists of a link imitating the quadriceps, a link simulating a tendon of the quadriceps and a link specifying the distance between the quadriceps and the femur. This subsystem can control the motion of the knee joint model. The patella subsystem (Fig.9) includes a link simulating the patella, patellar ligament, and a link that provides the correct movement of the patella. The weight of ligaments and tendons is small in comparison to other structures of the knee joint. Despite this, the total weight of the patella and its ligament is $0.31 \mathrm{~kg}$. The radius of the patella will be assumed as equal to 0.023 
$m$. The tibia subsystem (Fig.10) simulates the tibia whose length is $0.4 \mathrm{~m}$ and weight equal to $3.75 \mathrm{~kg}$. The ligaments subsystem (Fig.11) imitates the anterior and posterior cruciate ligament. The movement along the plane of the tibia is possible due to the movement of links simulating the anterior cruciate ligament. In addition, the movement of these two links defines the principle of the system motion.

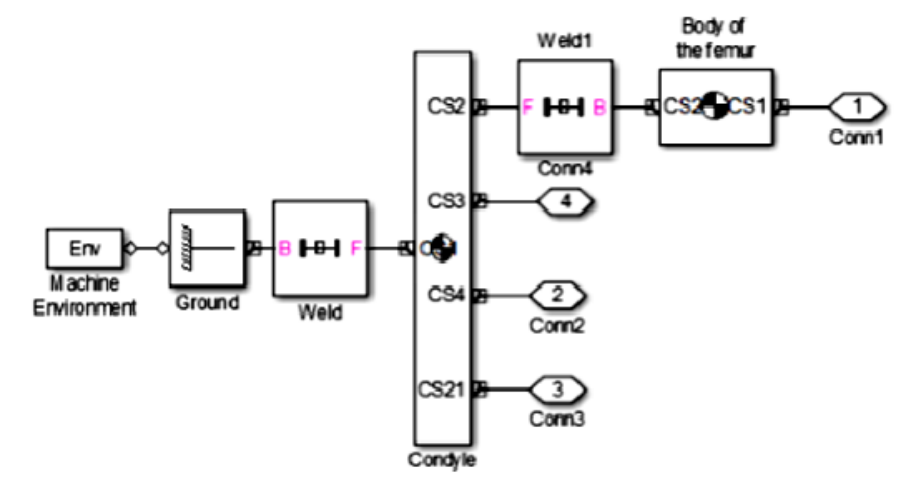

Fig.7. Femur subsystem.

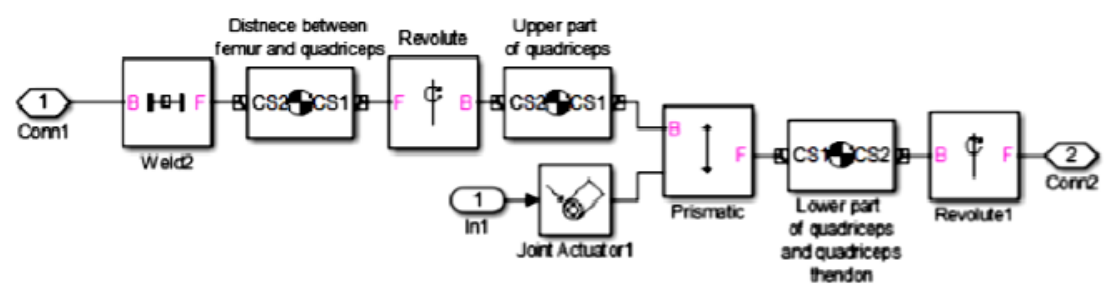

Fig.8. Subsystem Quadriceps.

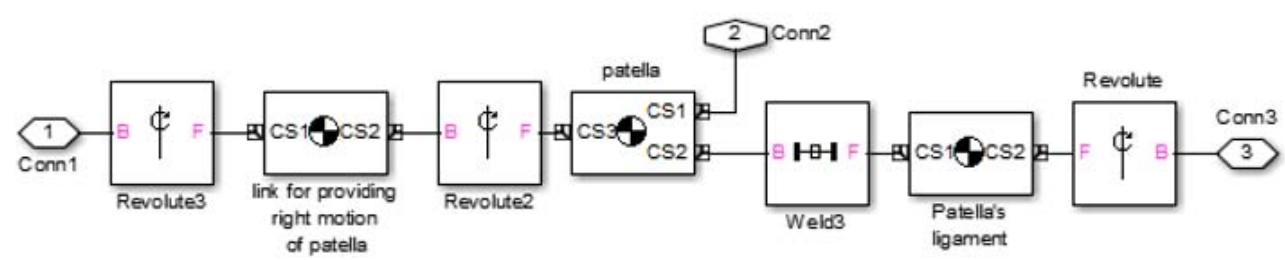

Fig.9. Subsystem Patella.

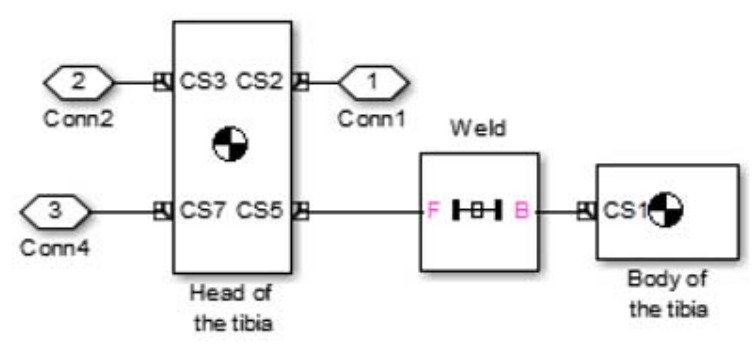

Fig.10. Tibia subsystem. 


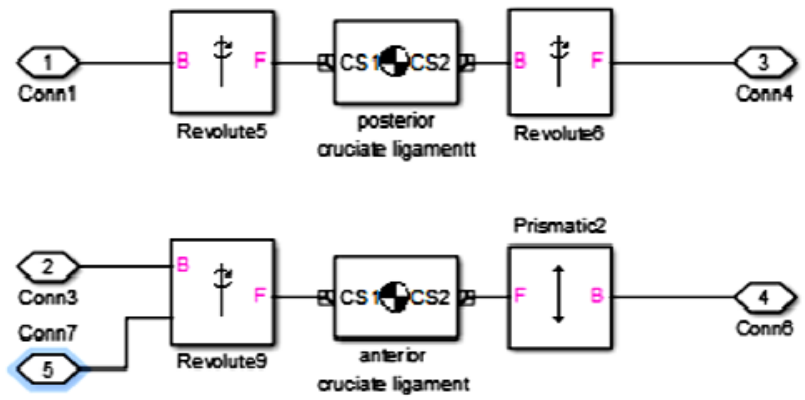

Fig.11. Ligaments subsystem.

\subsection{Model of the joint, supported by orthosis}

Assuming that the knee joint was damaged, the "circular ligament" links (links 6 and 7) were excluded. The modeled active orthosis provides only one degree-of-freedom.

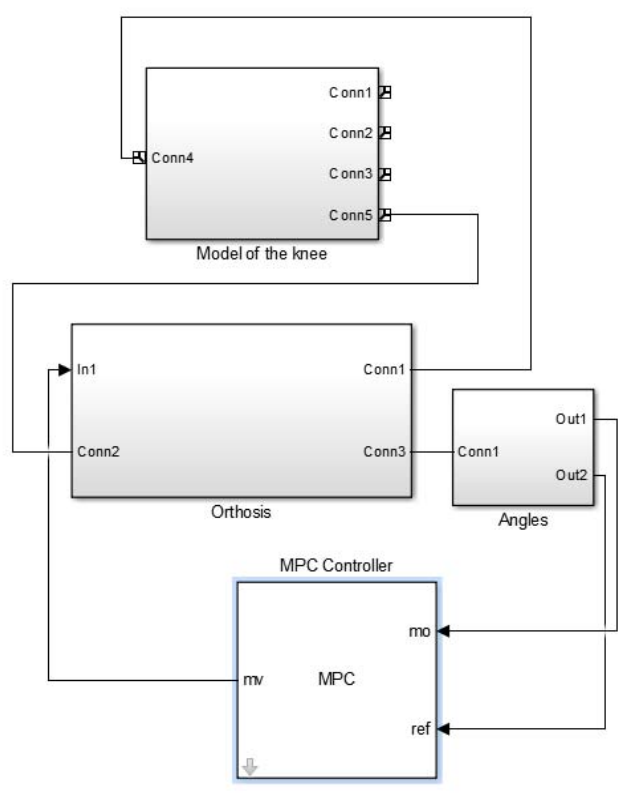

Fig.12. Model of the knee joint supported by orthoses.

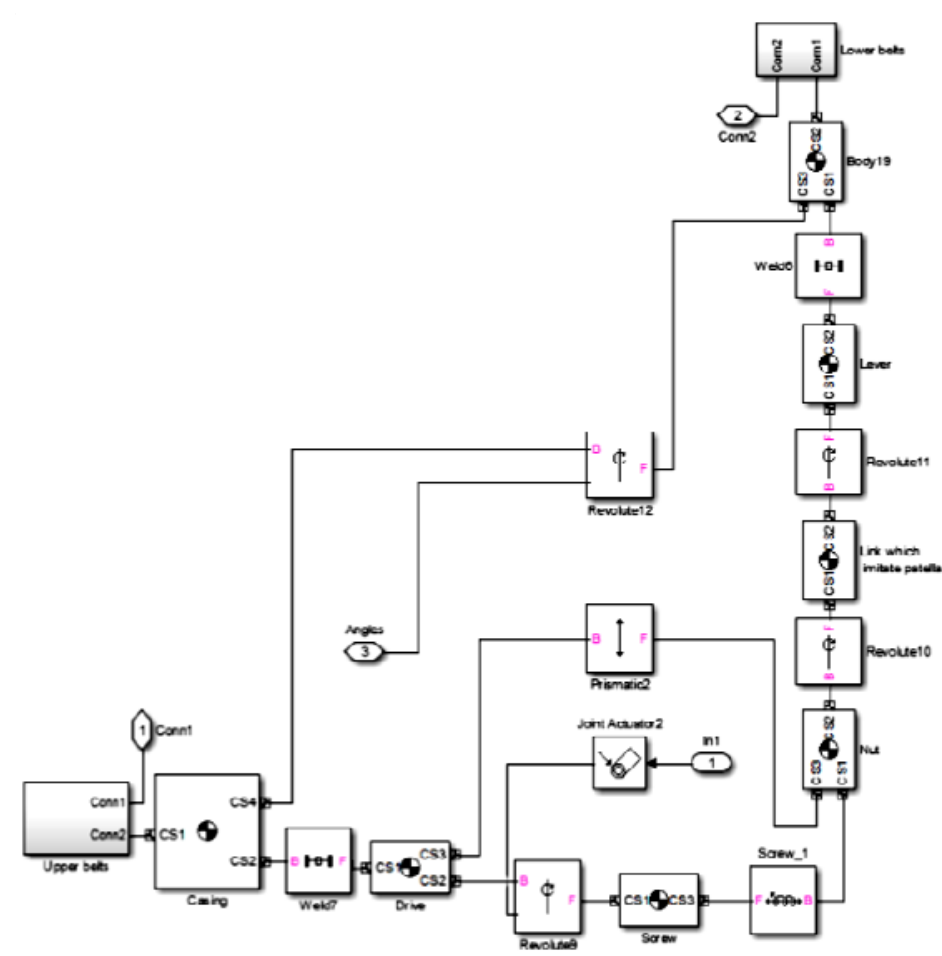

Fig.13. Subsystem orthosis model.

The model of the knee joint supported by the orthosis is illustrated in Fig.12. This model simulates motion of the knee joint during the gait where the MPC block controls the motion of the orthosis. The subsystem orthosis model (Fig.13), includes all the key aspects of the orthosis structure, such as fixing belts, ball screw pair, etc. 


\section{Knee-joint smart orthosis}

\subsection{Work principle}

The flexion of the lower part of the orthosis takes place due to the linear movement of the stock (4) that is connected to the motor-reducer by a ball-screw (BS) and a cylindrical gear. The prototype design is shown in Fig.14 [38].

The ball-screw allows converting a relatively small torque produced by the engine in a significant linear force, necessary to lift the person weight. Moreover, when using the ball-screw, its high precision, resistance to large axial loads, and self-locking ability are useful. In this kind of devices it is recommended to use ball-screws with four contact-points [39].

The stock (4) fixed to the BS nut moves in sliding pairs along the guide rails and is connected by a flat joint with the element, tentatively called "patella" (6), which allows a change of the force arm and a significant reduction of the pulling force acting on the ball-screw. The "patella" is connected to the lower half of the orthosis by a flat joint (7) and, rotating around its axis, produces flexion/extension of the limb. Belts (8) can be displaced along the shank for the optimal fitting of the orthosis. Using a telescopic element located between the "knee" and the "ankle" joints, it is possible to change the length of the orthosis in a range of $70 \mathrm{~mm}$. A rigid support between the "ankle" and the "heel support" renders it possible to minimize vertical load on the injured joint and can be removed in case of uselessness.

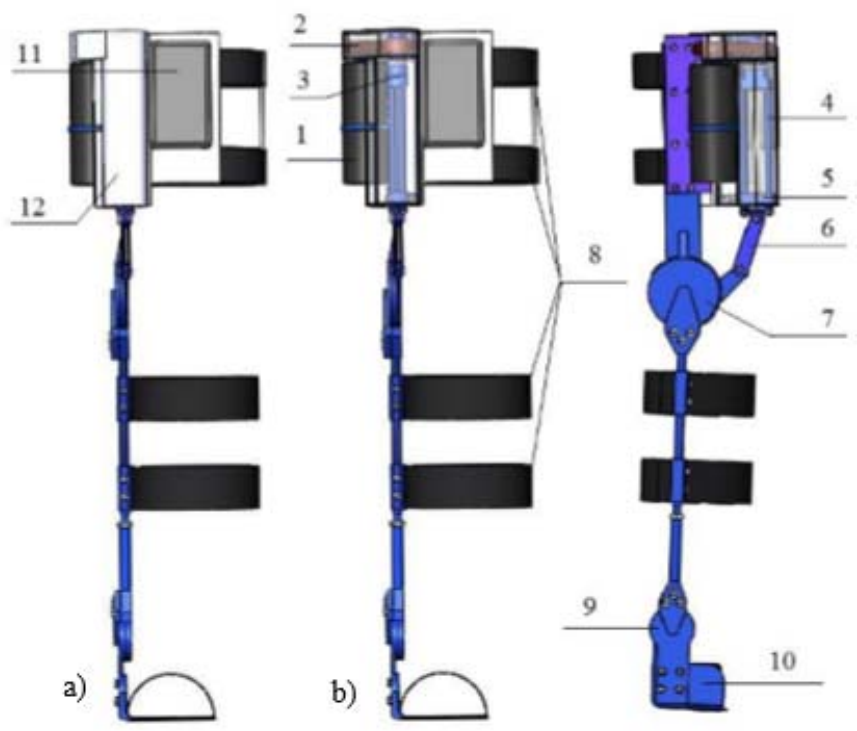

Fig.14. Design of the orthosis prototype. a) with protective casing, b) without protective casing. 1 servomotor 2 - gears, 3 - ball-screw, 4 - stock, 5 - joint, 6 - "patella", 7 - flat "knee" joint, 8 fixing belts with electrodes, 9 - flat "ankle" joint, 10 - "heel-support", 11 - controlling module, 12 protective casing.

\subsection{Loads and orthosis components}

To explore the walking process, a series of experiments was made using the Vicon system. The results showed that the walking process can be divided into a support phase and transfer phase. During the support phase, the full weight of the human body is applied only on one leg, but the leg is straight with no bending moments. During the transfer phase, the knee flexion angle does not exceed $30^{\circ}$ and the orthosis transfers only the weight of the shin. 


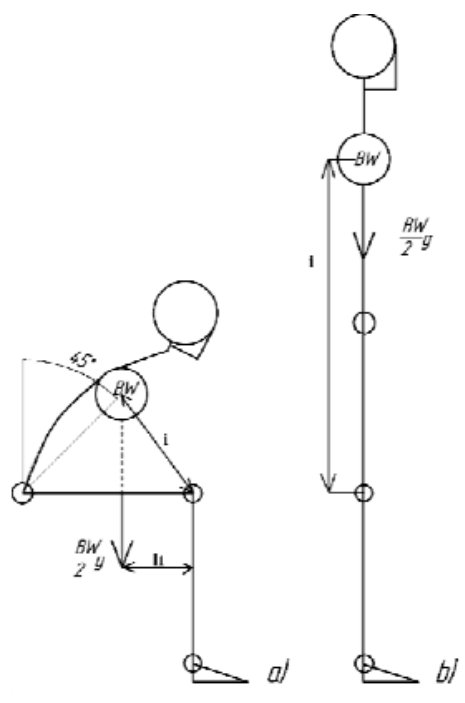

Fig.15. Position of gravity center.

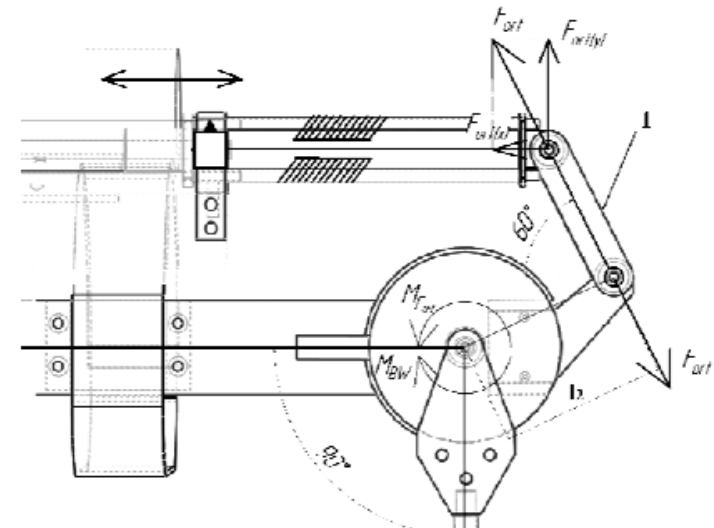

Fig.16. Scheme of static forces.

The maximum load will be applied on the orthosis at sit-to-stand motion. It is known that during standing up the quadriceps of each leg makes an effort to lift only half of the body weight excluding the mass of legs and feet. In the sitting position (Fig.15a), the center of gravity is located at a distance of about 200 $\mathrm{mm}$ from the axis of the knee joint. The scheme of load distribution in the orthosis is shown in Fig.16. On the basis of this, it is possible to calculate the necessary torque, taking into account the mass of an average person.

\section{Results of simulations}

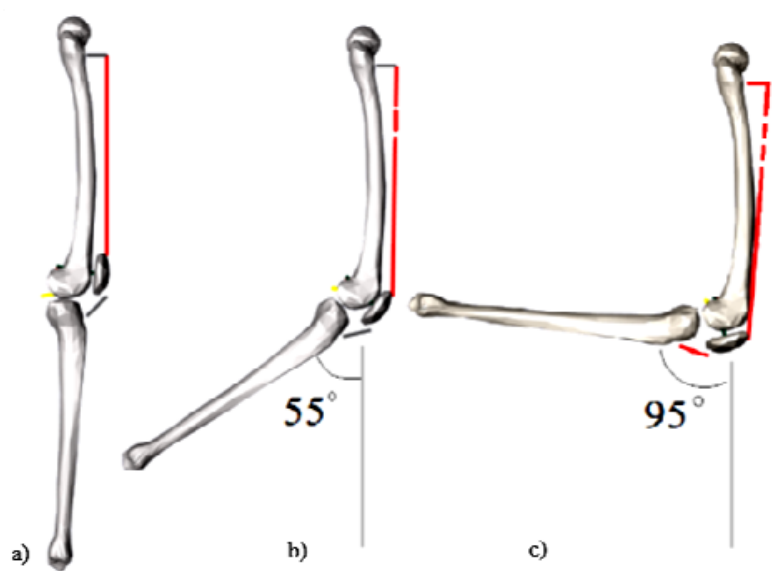

Fig.17. Model that imitates a healthy knee joint.
a) starting position,
b) maximal angle of flexion during the gait,
c) flexion of the knee joint.

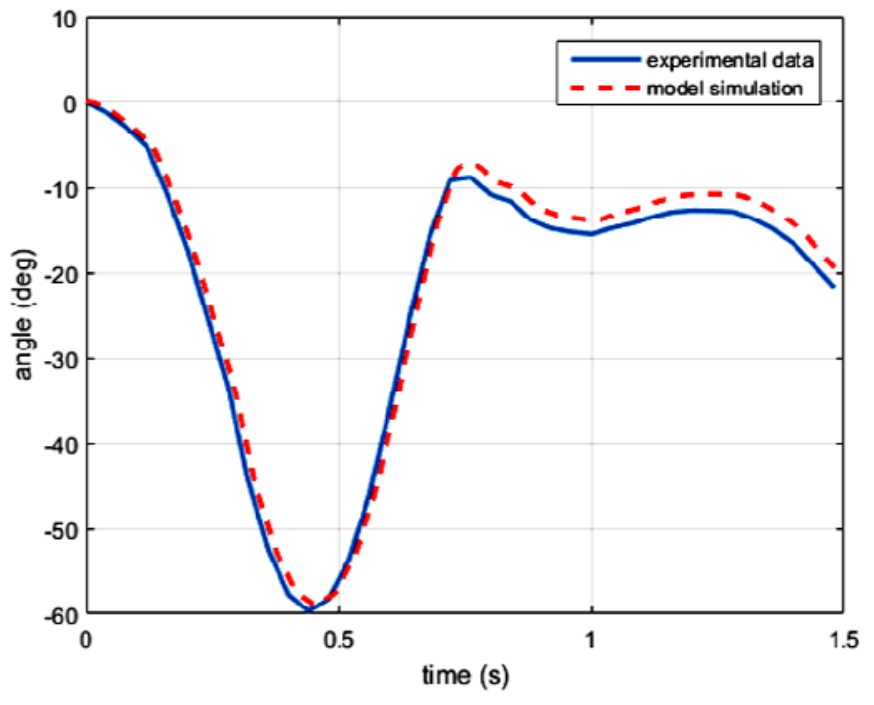

Fig.18. Movement of a healthy knee joint. 


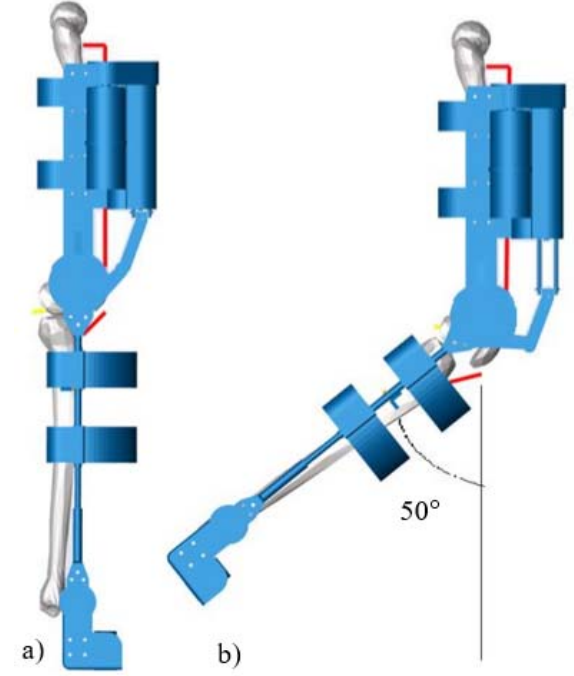

Fig.19. Model that imitates a healthy knee joint.

a) starting position,

b) maximal angle of flexion during the gait.

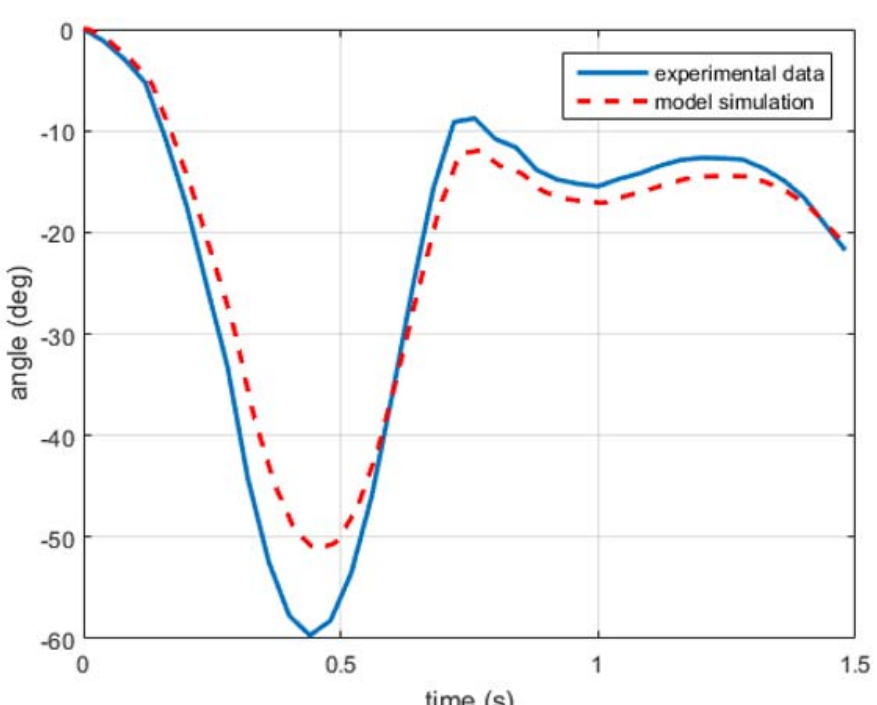

Fig.20. Movement of the knee joint supported by the orthosis.

The results of the simulation of a healthy knee joint were compared to data of real human gait [40] obtained by the motion capture system (Vicon). The working model simulating the motion of the healthy knee joint is shown in Fig.17. The comparison of the simulation results and the results obtained by the capture system is presented in Fig.18. The working model simulating motion of the injured knee joint supported by the orthosis is shown in Fig.19. The comparison of simulating results and the results obtained by the capture system is presented in Fig. 20.

In both cases the angle between the tibia and the femur during human gait was analyzed. The blue lines show the angle change obtained with the use of the motion capture system Vicon and the red lines show the simulation results. The correlation between experimental and simulation results is good enough. The obtained results are close enough in both cases. The correlation results of the analysis of the healthy knee joint (Fig.18) are better because, in this case, during tests and simulations, the same healthy joint was considered. In case of an injured knee joint (Fig.20), a joint supported by the orthosis was simulated and the obtained results were compared to the movement of a healthy knee joint. Because of this, the correlation of these results is worse but the maximum deviation is not more than $15 \%$.

\section{Model for controlling of the knee joint orthosis}

One of the most advantageous solutions for controlling different types of assistive prostheses is EMG technology.

Surface electrodes attached to the human skin can register and evaluate the electrical potentials produced by skeletal muscle cells when these cells are electrically or neurologically activated. The received EMG signals which are produced by skeletal muscles can be processed depending on their potential. Since the received impulses have different amplitudes, it is possible to register detailed necessary impulses and to evaluate them with a controller, which controls the servomotors in the exoprosthetics system.

The controlling assembly that is attached to the knee joint orthosis (Fig.14) consists of a high input impedance amplifier, which is connected to electrodes (8) attached to the human skin, an anti-aliasing filter, a microcontroller which digitizes and processes the EMG signals, a communication module sending the processing results to the processing unit which controls the servomotor (1).

This device is attached to the knee joint orthosis and to the human leg by adjustable belts that can be adjusted to all users. The surface electrodes are attached onto the skin surface to get the electrical potential 
generated by muscle cells when these cells are neurologically activated. The recorded EMG signals from the muscles of the limb were used for extracting kinematic variables (i.e., joint angles) in order to control the knee joint orthosis in real time. When the device detects electrical potentials produced by skeletal muscle cells, the controller sends the processing results to the processing unit, which controls the servomotor of artificial limbs. A standard machine learning method (namely, a Support Vector Machine) is used to build a point-to-point map between muscle activity and leg position/orientation/grasping force. The map relies on gravity compensation and rather slow movement speed, which enforces a many-to-one relationship between the EMG signals and the position [41] - [44].

\section{Conclusions}

1. The simulation results obtained by the model have a good enough correlation with the results obtained by measuring the real motion. Some errors can be caused by differences in masses and lengths of the analyzed links.

2. Despite these mistakes, it is possible to state that the designed orthosis can simulate the motion of the healthy knee joint on the sagittal plane. It means that the orthosis can be used and is advisable in the post traumatic period.

3. The future research steps can be as follows:

- Minimizing modelling mistakes,

- Model visualization,

- Simulating and testing different types of knee joints supported by active orthoses,

- Exploring different fixing points of the orthosis,

- Exploring the correct positioning of the orthosis and finding a suitable power source.

\section{Nomenclature}

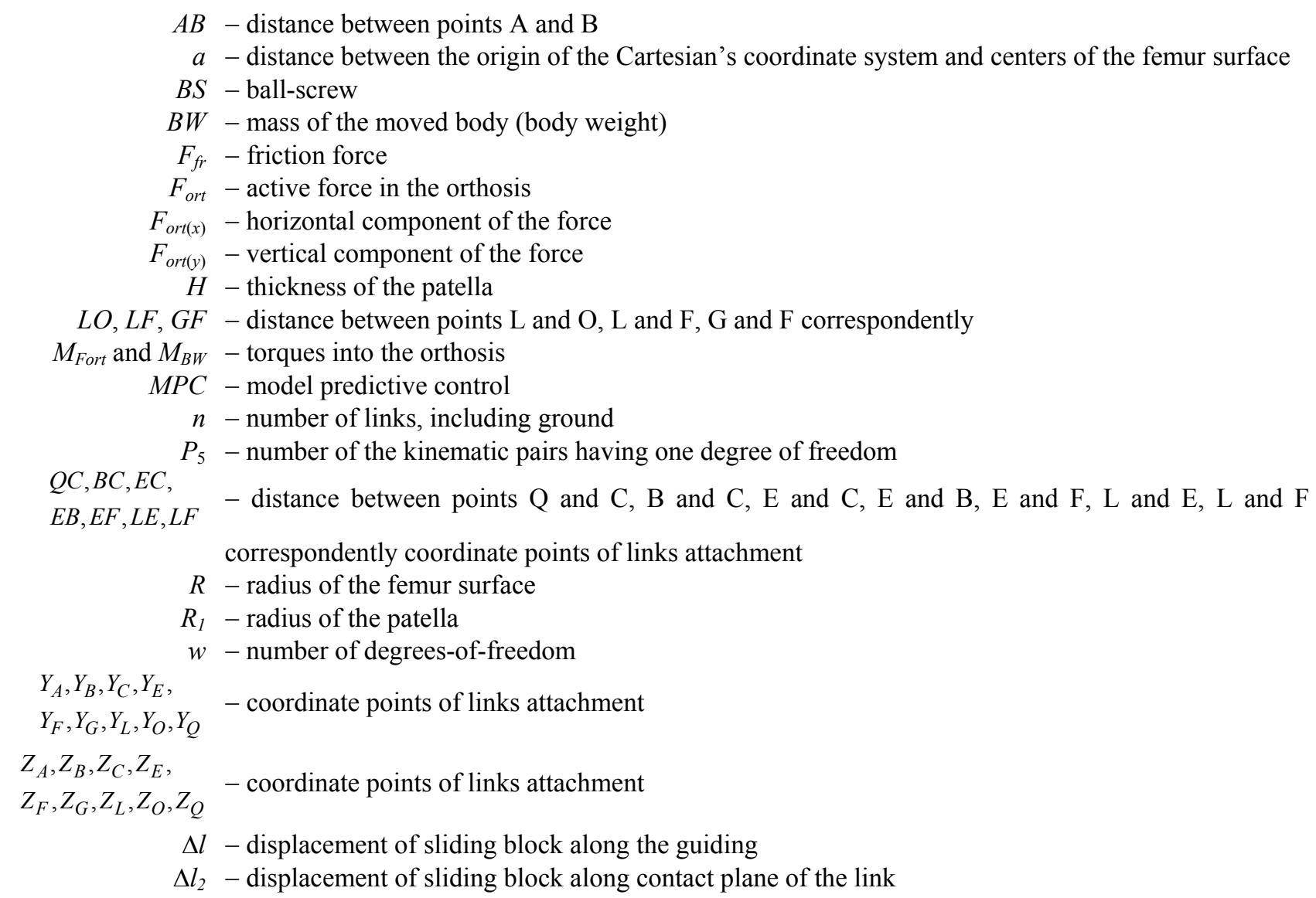




\section{Acknowledgements}

This research work has been supported by the School of Engineering (TTU) and by the Estonian Archimedes Foundation (project „Doctoral School of Energy and Geotechnology“).

\section{References}

[1] Saranli U., Arslan O., Ankarali M. and Morgul O. (2010): Approximate analytic solutions to nonsymmetric stance trajectories of the passive springloaded inverted pendulum with damping. - Nonlinear Dynamics, vol.62, No.4, pp.729-742.

[2] Kuo A. (2007): The six determinants of gait and the inverted pendulum analogy: A dynamic walking perspective. Human Movement Science, vol.26, pp.617-656.

[3] Kajita S., Kanehiro F., Kaneko K., Yok K. and Hirukawa H. (2001): The 3D linear inverted pendulum model: $A$ simple modeling for a biped walking pattern generation. - International Conference on Intelligent Robots and Systems, pp.239-246.

[4] Otani T., Hashimoto K., Yahara M., Miyamae S., Isomichi T., Hanawa S., Sakaguchi M., Kawakami Y., Lim H. and Takanishi A. (2015): Utilization of human-like pelvic rotation for running robot. - Frontiers in Robotics and AI, vol.2 (Article 17), pp.1-9.

[5] Pamies R. (2012): Application of Multibody Dynamics Techniques to the Analysis of Human Gait. - Ph.D. Dissertation, Polytechnic University of Catalonia.

[6] Lim H., Ogura Y. and Takanishi A. (2008): Locomotion pattern generation and mechanisms of a new bipedwalking machine. - Royal Society, Proceeding A, vol.464 (2089), pp.273-288.

[7] Chigarev A. and Borisov A. (2010): Simulation of controlled motion of the bipedal anthropomorphic mechanism. Russian Journal of Biomechanics, PNIPU: 74-88.

[8] Zhang L., Brunnett G. and Rusdorf S. (2011): Real-time Human Motion Capture with Simple Marker Sets and Monocular Video. - Journal of Virtual Reality and Broadcasting, vol.8, No.1.

[9] Dodd K., Morris M., Matyas T., Wrigley T. and Goldie P. (1988): Lateral pelvic displacement during walking: retest reliability of a new method of measurement. - Gait \& Posture, vol.7, No.3, pp.243-250.

[10] Galdeano D., Bonnet V., Bennehar M., Fraisse P. and Chemori A. (2012): Partial Human Data in Design of Human-Like Walking Control in Humanoid Robotics. - 10th IFAC Symposium on Robot Control, Syroco, Dubrovnik, Croatia.

[11] Ito T. (2009): Walking motion analysis using small acceleration sensors. - International Journal of Simulation: Systems, Science and Technology, vol.10, No.3, pp.65-71.

[12] Tanaka S., Motoi K., Nogawa M. and Yamakoshi K. (2004): A new portable device for ambulatory monitoring of human posture and walking velocity using miniature accelerometers and gyroscope.- 26th Annual International Conference of the IEEE, Engineering in Medicine and Biology Society, vol.1, pp.2283-2286.

[13] Cross R. (1999): Standing, walking, running, and jumping on a force plate. - American Journal of Physics, vol.67, No.4, pp.304-309.

[14] Marasovic T., Cecic M. and Zanchi V. (2009): Analysis and interpretation of ground reaction forces in normal gait. - WSEAS Transactions on Systems, vol.8, No.6, pp.1105-1114.

[15] Yorozu A., Moriguchi T. and Takahashi M. (2015): Improved leg tracking considering gait phase and spline-based interpolation during turning motion in walk tests. - Sensors, vol.15, No.9, pp.22451-22472.

[16] de Araújo V., Alsina P., Soares L. and de Medeiros A. (2012): Generation of Anthropomorphic movements for an active orthosis for lower limbs. - ABCM Symposium Series in Mechatronics, vol.5 (Section VII - Robotics), pp.1052-1057. 
[17] Zhigailov S., Musalimov V. and Aryassov G. (2014): Design of experimental stand for human gait imitation. Proceedings of the International Conference of DAAAM Baltic „Industrial Engineering“, pp.300-304.

[18] Zhigailov S., Kuznetcov A., Musalimov V. and Aryassov G. (2015): Measurement and analysis of human lower limbs movement parameters during walking. - Solid State Phenomena, vol.220-221, pp.538-543.

[19] Gayvoronskiy I.V. (2011): Anatomy and physiology: textbook for students. Institutions of prof. education. Publishing Center "Academy", Moscow.

[20] Krokhaleva N. (2015): Modeling of the working surfaces geometry of the knee joint elements. - Closing Conference of the Project "Doctoral School of Energy and Geotechnology II", Tallinn University of Technology, pp.203-204.

[21] http://bodybuilding.k21vek.com/anatomy, May 2017.

[22] Farhat N., Mata V., Rosa D., Fayos J. and Peirau X. (2009): Musculo-skeletic Model for Knee Joint Forces Estimation in Sport Activities. - 7th Euromech Solid Mechanics Conference J. Ambrósio et al. (eds.) Lisbon, Portugal.

[23] Dewen J., Ruihong Z., Ho D., Rencheng W. and Jichuan Z. (2003): Kinematic and dynamic performance of prosthetic knee joint using six-bar mechanism. - Journal of Rehabilitation Research and Development, vol.40, No.1, pp.39-48.

[24] Blankevoort L., Kuiper J., Huiskes R. and Grootenboer H. (1991): Articular contact in a 3-dimensional model of the knee. - Journal of Biomechanics, vol.24, No.11, pp.1019-1031.

[25] Halloran J., Petrella A. and Rullkoetter P. (2004): Explicit finite element modeling of total knee replacement mechanics. - Journal of Biomechanics, vol.38, No.2, pp.323-331.

[26] Catana M. and Tarnita D. (2013): Modeling, simulation and optimization of a human knee orthotic device. Applied Mechanics and Materials, vol.371, pp.549-553.

[27] Cyberdyne. http://www.cyberdyne.jp, May 2016.

[28] ReWalk. http://rewalk.com, May 2016.

[29] EksoBionics. http://intl.eksobionics.com, May 2016.

[30] Exoatlet. http://www.exoatlet.ru, May 2016.

[31] Sharkov O., Zolotov I. (2011): Influence of the geometric properties of an external housing on its stress-strain state. - Russian engineering research, vol.31, No.4, pp. $335-337$.

[32] Weinberg B., Nikitczuk J., Patel S., Patritti B., Mavroidis C., Bonato P. and Canavan P. (2007): Design, control and human testing of an active knee rehabilitation orthotic device. - IEEE International Conference on Robotics and Automation, pp.4126-4133.

[33] Pratt P. and Krupp B. (2004): The robokne: an eksoskeleton for enhancing strength and endurance during walking. - IEEE International Conference on Robotics and Automation, pp.2430-2435.

[34] Unluhisarcikli O. and Mavroidis C. (2013): Lower Extremity Exoskeleton For Gait Retraining. - United States Patent Application Publication.

[35] Human height. https://en.wikipedia.org/wiki/Human_height, May 2016.

[36] Determine humans' height by length of the long bones of the skeleton. http://www.forensmed.ru/tools/antr/ height.php, May 2016.

[37] Ovchinnikov I. (2016): Human Gait Modeling Using MPC Controller. - MSc. Dissertation, Tallinn University of Technology, Tallinn, Estonia.

[38] Turchinovich N. (2016): Design of Knee Joint Support System. - MSc. Dissertation, Tallinn University of Technology, Tallinn, Estonia.

[39] Penkov I. and Aleksandrov D. (2011): Axial displacements in ball screw mechanisms with two and four contactpoint. - International Review of Mechanical Engineering, vol.5, No.7, pp.1213-1218. 
[40] Krokhaleva N. (2016): Orthosis Supported Human Lower Limb Motion Model. - MSc. Dissertation, Tallinn University of Technology, Tallinn, Estonia.

[41] Vogel J., Castellini C. and van der Smagt P. (2011): EMG-Based Teleoperation and Manipulation with the DLR LWR-III. - IEEE/RSJ International Conference on Intelligent Robots and Systems. September 25-30, San Francisco, CA, USA.

[42] Wang J., Tang L. and Bronlund J. Surface EMG Signal Amplification and Filtering. - IEEE/RSJ International Journal of Computer Applications, 0975 - 8887.

[43] Stepp C., Dellon B. and Matsuoka Y. (2010): Contextual effects on robotic experiments of sensory feedback for object manipulation. - IEEE RAS \& EMBS International conference on Biomedical Robotics and Biomechatronics, Tokyo, pp.58-63.

[44] Cavallaro E., Rosen J., Perry J., Burns S. and Hannaford B. (2005): Hillbased model as a myoprocessor for a neural controlled powered exoskeleton arm - parameters optimization. - IEEE International Conference on Robotics and Automation, Barcelona, Spain. 